\title{
FURTHER STUDIES ON THE EPIDEMIC ABORTION REACTIONS IN CHILDREN *
}

\author{
J. P. SEDGWICK, M.D. \\ Associate Professor in Charge of the Division of Pediatrics \\ AND \\ W. P. LARSON, M.D. \\ Assistant Professor of Bacteriology, University of Minnesota \\ MINNEAPOLIS
}

In a previous paper ${ }^{1}$ we called attention to the fact that the blood serums of children consuming milk from cattle infected with contagious abortion often contain antibodies against Bacillus abortus.

Contagious abortion of cattle has been known to stockmen and dairymen the world over for the past century or more. There is probably no disease or combination of diseases which distresses the dairyman more or causes greater economic loss to him than this particular infection.

Up to the year 1896 the etiology of this disease remained obscure. In that year Dr. Bang ${ }^{2}$ of Copenhagen isolated the organism which is now recognized as the specific etiologic agent in this disease. It is a short gram-negative, nonmotile bacillus which is somewhat diffcult to isolate, but when once it has been grown successfully on artificial mediums its further cultivation presents few difficulties. The work of Bang shows that this bacillus is not only infectious for cattle, but for almost all of the domestic and laboratory animals. Ostertag found that it often caused abortion in mares.

In the domestic animals the site of infection appears to be in the uterine mucosa of pregnant animals. Until within the last two or three years the uterine mucosa was thought to be the only site of infection. Through the excellent work of Smith, ${ }^{3}$ Fabyean, ${ }^{4}$ Schroeder and Cotton $^{5}$ we know that the Bacillus abortus will affect laboratory animals and that abortion is not the only sign of infection. These authors have found that when a guinea-pig is inoculated intraperitoneally an infection occurs which cannot be differentiated macroscopically from tuberculosis. The spleen, especially, becomes very much enlarged and presents an appearance similar to that in an animal infected with tuberculosis. Not only the spleen and lymphatic system,

\footnotetext{
* Read before the Section on Diseases of Children at the Sixty-Sixth Annual Session of the American Medical Association, San Francisco, June, 1915.

1. Larson and Sedgwick: Am. Jour. Dis. ChILD., 1913, vi, 326.

2. Bang: Ztschr. f. Tiermed., 1897.

3. Smith and Fabyean: Centralbl. f. Bakt. Referate, 1908, 1xi, 549.

4. Fabyean: Jour. Med. Research, 1912, xxi, 441.

5. Schroeder and Cotton: Bull. Bureau of Animal Industry, 1912.
} 
but the bones, liver, and kidneys have likewise been found to be extensively involved.

We have had opportunity in the last two or three years to go over the work of Smith, Fabyean, Schroeder and Cotton and can confirm the work of these authors in every particular. Laboratory animals that become infected do not always abort. In fact, we have had animals which showed the lesions above described in very pronounced degree carry to term. It thus appears that the type of lesion produced by the Bacillus abortus depends entirely on the animal infected.

Owing to the prevalence of this disease among cattle, it would be logical to expect to find that this organism is widely disseminated in market milk. From the excellent work of the Bureau of Animal Industry we know that a very large percentage of market milk contains this organism.

Before the work of the authors above referred to was made public, the question had raised itself with us as to the possibility of children who consumed milk from infected herds being infected. In our first series of 425 children whose blood was tested to either or both agglutination and complement-fixation reactions, we found that 17 per cent. of our cases gave a positive reaction. The further interesting fact was observed that children known to be supplied with milk from a perfectly clean herd, that is, one which had never been affected with contagious abortion, did not give this reaction, while those fed on milk purchased on the market gave a high percentage of positive reactions.

We were able to show by specific absorption tests that we were dealing with true antibodies against the Bacillus abortus, as a positive serum could readily be rendered negative by depleting it of its antibodies by absorption with the Bacillus abortus. The significance of these findings we were not able to determine; whether the presence of these antibodies is the result of an infection of the child or the result of antibody absorption through the digestive tract, cannot at present be definitely decided. While there is no positive proof that the reactions are due to an infection of the child, we are inclined to accept this interpretation. While it has been shown that antibodies are excreted in milk and that these may be absorbed through the intestinal canal, it would hardly seem probable that these antibodies could be excreted in the milk and absorbed by the child through the intestinal tract in sufficient quantities to give a positive complement-fixation and agglutination reaction. Bang has shown that animals may become infected by the intestinal tract just as readily and almost with the same degree of certainty as when the organisms are injected subcutaneously or intravenously. If the same is true of human beings, it will be readily seen how anyone who consumes milk is amply exposed to infection. 
As we mentioned in our earlier contribution, we have found that women who abort do at times give a complement-fixation reaction when the Bacillus abortus is used as an antigen. In the bloods of aborting women examined by Iarson this reaction was even more common than the Wassermann reaction. We are indebted also to Dr. A. W. Robertson of Litchfield, Minn., for the report of two women who aborted during the time that there was an epidemic of abortion among the cattle on their farms. In these cases he was able to find no other causes by which he could explain the expulsion of the fetuses. Although these reports are not yet sufficient to form a basis for definite conclusion, they are certainly suggestive.

Although our earlier 425 cases showed that 17 per cent. of the children gave a positive fixation and agglutination test for the Bacillus abortus, the children in some groups gave a much higher proportion, in fact over 40 per cent. of the children in one institution gave positive reactions and one group gave as high as 48 per cent.

It appeared to us that the next step in attacking this problem was the determination as to whether new-born children, who had as yet received no cow's milk, give this reaction. In a series of new-born children at the Minnesota University Hospitals the blood was collected from the cord at birth, or from the child's heel during the first few days after birth, and immediately examined. In all, 42 such new-born children were examined, without a single positive reaction. Eighteen of the above forty-two children were girls. Three of the above infants died and postmortem examinations showed:

1. Baby $H .:$ Bronchopneumonia with cardiac dilatation.

2. Baby N.: Congenital syphilis and bronchopneumonia. This child gave a positive Wassermann and a negative abortus reaction.

3. Baby $\mathrm{K}$ : : Bronchopneumonia and congenital hydronephrosis.

Baby boy $\mathrm{Cl}$., who was not included in the above series, as he received other feeding than breast milk, is exceptionally interesting. His blood gave a positive reaction using the Bacillus abortus as antigen. He was taken from the breast on the seventh day after birth as he had a high fever, the cause of which was not ascertained. He was then fed on artificial food containing cow's milk and on the twentyfirst day the reaction was positive. This is the earliest positive bacillus abortion reaction which we have observed, and it is especially to be noted that the reaction was found two weeks after removal from the breast. The child recovered.

As an enlarged spleen is one of the findings that is observed in laboratory animals which have been infected with the Bacillus abortus, we were especially on the lookout for positive reaction in children with clinically demonstrable enlargement of this viscus. 
Baby B. gave a positive reaction and had an enlarged spleen. It was 19 months old, with fair nutrition and had been on a general diet. The postmortem examination showed empyema, abscess of the lung, suppurative coxitis and adenitis.

Gladys K., who had an enlarged spleen, gave a complete inhibition.' She was 10 years old and fairly well nourished. She was on a general diet. Her previous illness had been tonsillitis and pertussis. She was in the hospital for epilepsy, but gave at times very marked spasmophilic reactions.

Baby B., 2 months old, with pneumonia and an enlarged spleen, gave a negative reaction.

Ger, a girl 18 months old, with rickets and an enlarged spleen, also gave a negative reaction.

As this reaction is carried out in the same manner as the Wassermann test except that the Bacillus abortus is used as an antigen, the fact that the blood of five children with congenital syphilis gave negative reactions with this test should be recorded.

As bone lesions suggestive of rickets have been produced in laboratory animals by injection of the Bacillus abortus, the fact that several marked cases of rickets gave negative reactions is of interest.

Other conditions in which negative reactions were found were eczema, hydrocephalus, nephritis, atrophy, varicella, measles, diphtheria, bronchopneumonia, chorea, enteritis, anemia, imbecility and chronic pulmonary tuberculosis.

\section{SUMMARY}

1. This further study confirms our earlier report of the finding of a deviation of complement in children with the use of the Bacillus abortus as antigen.

2. New-born children who have been fed at the breast only, do not show the reaction. One baby, 3 weeks old, after having been off the breast two weeks and on a food containing cow's milk, gave a positive reaction.

3. The finding of positive reactions in children with large spleens is suggestive.

4. The blood of children giving positive Wassermann reactions may show no deviation of complement when the Bacillus abortus is used as antigen.

5. Children with many of the common diseases of children, including rickets, gave negative reactions. Much further study is, however, necessary with such conditions.

6. For the practicing physician, the importance of breast feeding and the desirability of the heating of all cow's milk administered is emphasized. 